\title{
Organ Craniocaudal Length Measurement
}

National Cancer Institute

\section{Source}

National Cancer Institute. Organ Craniocaudal Length Measurement. NCI Thesaurus.

Code C135509.

The linear extent along the craniocaudal axis from one end of an organ to the other end. 